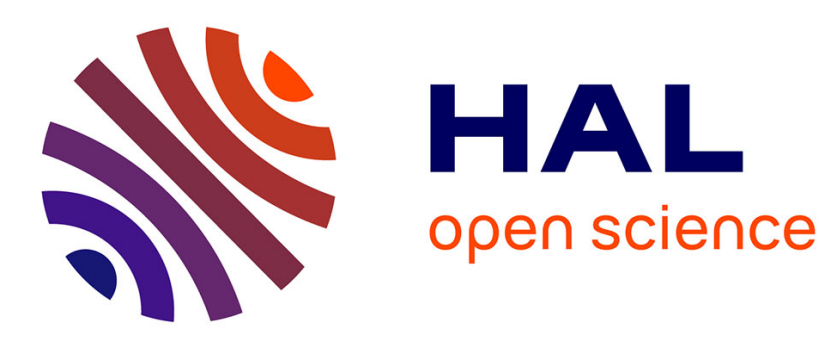

\title{
The role of solvent swelling in the self-assembly of squalene based nanomedicines $\dagger$
}

Debasish Saha, Fabienne Testard, Isabelle Grillo, Fatima Zouhiri, Didier

Desmaele, Aurel Radulescu, Sylvain Desert, Annie Brulet, Patrick Couvreur, Olivier Spalla

\section{To cite this version:}

Debasish Saha, Fabienne Testard, Isabelle Grillo, Fatima Zouhiri, Didier Desmaele, et al.. The role of solvent swelling in the self-assembly of squalene based nanomedicines $\dagger$. Soft Matter, 2015, 11, pp.4173. 10.1039/c5sm00592b . hal-01228690

\section{HAL Id: hal-01228690 https://hal.science/hal-01228690}

Submitted on 17 Nov 2015

HAL is a multi-disciplinary open access archive for the deposit and dissemination of scientific research documents, whether they are published or not. The documents may come from teaching and research institutions in France or abroad, or from public or private research centers.
L'archive ouverte pluridisciplinaire HAL, est destinée au dépôt et à la diffusion de documents scientifiques de niveau recherche, publiés ou non, émanant des établissements d'enseignement et de recherche français ou étrangers, des laboratoires publics ou privés. 


\title{
Soft Matter
}

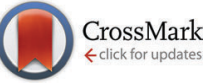

Cite this: Soft Matter, 2015 11, 4173

Received 12th March 2015, Accepted 7th April 2015

DOI: $10.1039 / \mathrm{c} 5 \mathrm{sm} 00592 \mathrm{~b}$

www.rsc.org/softmatter

\section{The role of solvent swelling in the self-assembly of squalene based nanomedicines $\dagger$}

\author{
Debasish Saha, ${ }^{a}$ Fabienne Testard, ${ }^{a}$ Isabelle Grillo, ${ }^{b}$ Fatima Zouhiri, ${ }^{c}$ \\ Didier Desmaele, ${ }^{c}$ Aurel Radulescu, ${ }^{d}$ Sylvain Desert, ${ }^{e}$ Annie Brulet, ${ }^{e}$ \\ Patrick Couvreur ${ }^{c}$ and Olivier Spalla*a
}

\begin{abstract}
Squalene based nanoparticles obtained via nanoprecipitation are promising candidates as efficient anticancer drugs. In order to highlight their preparation process and to facilitate further clinical translation, the present study enlightens the paramount role of the solvent in the formation of these nanomedicines. Three different squalene-based nanoparticles, i.e. squalenic acid, deoxycytidine squalene and gemcitabine squalene, have been investigated before and after organic solvent evaporation. Size and structural analysis by Small Angle Neutron Scattering revealed that droplets' size was uniquely controlled by the solvent composition (ethanol-water), which evolved during their gradual formation. The particles were preferably swollen by water and the swelling increased when less ethanol was present. Either coalescence or fragmentation was observed depending on the increase or decrease of the ethanol content, supporting an equilibrium control of the size. Moreover, a high water swelling was observed for the three local organization of the nanodroplets (hexagonal for gemcitabine squalene, cubic for deoxycytidine and not structured for squalenic acid) and could be the source of the previously reported efficiency of related anti-cancer squalene based nanomedicines.
\end{abstract}

\section{Introduction}

Increasing the amount of therapeutic agent reaching the desired biological target is one of the key points motivating all the present endeavors in nanomedicine. In this context, various nucleoside analogues with anticancer or antiviral pharmacological activity have been conjugated to squalene, a natural and biocompatible lipid (i.e., squalenoylation technology). The resultant conjugates were found to display an amphiphilic character and a precipitation method by solvent displacement provided the spontaneous formation of nanoparticles in water. ${ }^{1,2}$ Among others, this concept has been applied to gemcitabine, an anticancer agent, prescribed in first intention for the pancreatic cancer. ${ }^{3}$ It was observed that the so-called squalene-gemcitabine nanoparticles (SQ-gem NPs) were able to inhibit gemcitabine

\footnotetext{
${ }^{a}$ CEA Saclay, DSM/IRAMIS/NIMBE/LIONS, UMR CEA/CNRS 3299, 91191 Gif sur Yvette, France. E-mail: Olivier.spalla@cea.fr

${ }^{b}$ Institut Laue-Langevin, 38042 Grenoble Cedex 9, BP 156, France

${ }^{c}$ Université Paris-Sud, UMR CNRS 8612, Faculté de Pharmacie, Châtenay-Malabry, F-92296, France

dJülich Centre for Neutron Science JCNS, Forschungszentrum Jülich GmbH, Outstation at MLZ, Lichtenbergstraße 1, 85747 Garching, Germany

${ }^{e}$ Laboratoire Léon Brillouin UMR12 CEA-CNRS, Bât 563 CEA Saclay, 91191 Gif sur Yvette Cedex, France

$\dagger$ Electronic supplementary information (ESI) available. See DOI: 10.1039/ c5sm00592b
}

metabolization in human plasma, to increase the absorption in lymphoid organs ${ }^{4}$ after both intravenous and oral administration and to prolong the concentrations of gemcitabine in the plasma after intravenous administration. ${ }^{5}$ In vitro and in vivo experiments have demonstrated an increased anticancer activity in experimental models of leukemia ${ }^{6,7}$ and pancreatic cancers ${ }^{8}$ and these nanoparticles were even found to overcome some mechanism of resistance, ${ }^{9}$ including the down regulation of nucleoside transporters or the insufficient phosphorylation by deoxycytidine kinase. One of the most intriguing aspects of this efficient nanomedicine results from the spontaneous selfassembly of the gemcitabine-squalene prodrug into nanoparticles. The unique property of squalene to adopt a dynamically folded molecular conformation in aqueous media is likely a key, but the mechanism allowing the formation of these nanoparticles, as well as, the events occurring during the nanoprecipitation of the SQ-gem bioconjugate remained unclear and deserved to be investigated. This is a very important question, because of the translation from the bench to the bed side and the design of a clinical sample needs a better understanding of nanoparticle elaboration. Thus, by using Small Angle Neutron Scattering (SANS), we investigated the formation process of the squalenoyl derivative nanoparticles, their size distribution, the solvent nanoparticle inner content and the nanostructure of gemcitabine-squalene (SQ-gem) and two other derivatives, the deoxycytidine-squalene (SQ-dC) nanoparticles and squalenic 
acid (SQ-CO2H) used as precursors in the synthetic procedure of squalenoyl compounds.

We observed drastic changes in the size, the swelling by solvent and the number density of particles along the gradual formation of SQ-dC and SQ-gem nanoparticles. These facts support the conclusion of a thermodynamically driven formation providing nanoparticles in equilibrium with their solvent environment. These results obtained in the field of squalenoylation technology impact the global understanding of the formation of prodrug nanoparticles ${ }^{10}$ and should help to reach a better control over size and stability of such very promising systems for enhanced drug activity and delivery.

\section{Experimental}

\subsection{Materials and characterization of squalene molecules}

Squalenic acid, deoxycytidine squalene and gemcitabine squalene (Fig. 1) were synthesized following the protocol described by Desmaele et al. ${ }^{1,11}$ The Table 1 summarizes the physical characteristics used for scattering calculation of the different squalenoyl derivatives. Absolute ethanol ( $>99.8 \%$ ) was purchased from Sigma-Aldrich, Germany and $\mathrm{D}_{2} \mathrm{O}(99.85 \%$ D) was purchased from Euriso-top.

\subsection{Preparation of nanoparticles (NPs)}

The nanoparticles were prepared by nanoprecipitation, which is a simple one-step method consisting of the addition of the squalene bioconjugate dissolved in an organic solvent to an aqueous phase. ${ }^{12-14}$ As presented in Fig. 2, this mixing step yields the intermediate states of nanoparticle formation. The organic

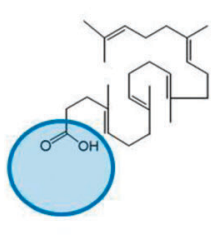

A

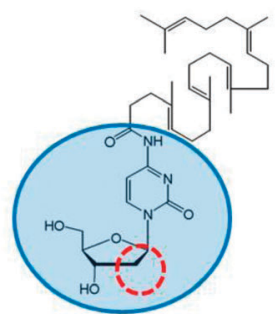

B

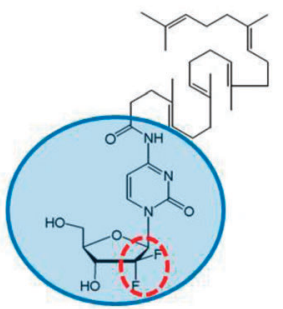

C
Fig. 1 Molecular structure of different squalenoyl molecules. (A) Deoxycytidine squalene (SQ-dC) (B) gemcitabine squalene (SQ-gem) (C) squalenic acid (SQ-CO2H).

Table 1 Characterization of different squalenoyl molecules in terms of their chemical formula, molecular weight, density and scattering length density

\begin{tabular}{lllll}
\hline $\begin{array}{l}\text { Squalenoyl } \\
\text { molecule }\end{array}$ & $\begin{array}{l}\text { Chemical } \\
\text { formula }\end{array}$ & $\begin{array}{l}\text { Molecular } \\
\text { weight } \\
\left(\mathrm{g} \mathrm{mol}^{-1}\right)\end{array}$ & $\begin{array}{l}\text { Density of } \\
\text { squalene } \\
\left(\mathrm{g} \mathrm{mL}^{-1}\right)\end{array}$ & $\begin{array}{l}\text { Scattering } \\
\text { length } \\
\text { density }\left(\mathrm{cm}^{-2}\right)\end{array}$ \\
\hline $\begin{array}{l}\text { Deoxycytidine } \\
\text { squalene }\end{array}$ & $\mathrm{C}_{36} \mathrm{H}_{55} \mathrm{~N}_{3} \mathrm{O}_{5}$ & 609.8 & & $7.70 \times 10^{9}$ \\
$\begin{array}{l}\text { Gemcitabine } \\
\text { squalene }\end{array}$ & $\mathrm{C}_{36} \mathrm{H}_{53} \mathrm{~N}_{3} \mathrm{O}_{5} \mathrm{~F}_{2}$ & 645.8 & 0.858 & $8.77 \times 10^{9}$ \\
Squalenic acid & $\mathrm{C}_{27} \mathrm{H}_{44} \mathrm{O}_{2}$ & 400.6 & & $3.42 \times 10^{9}$
\end{tabular}

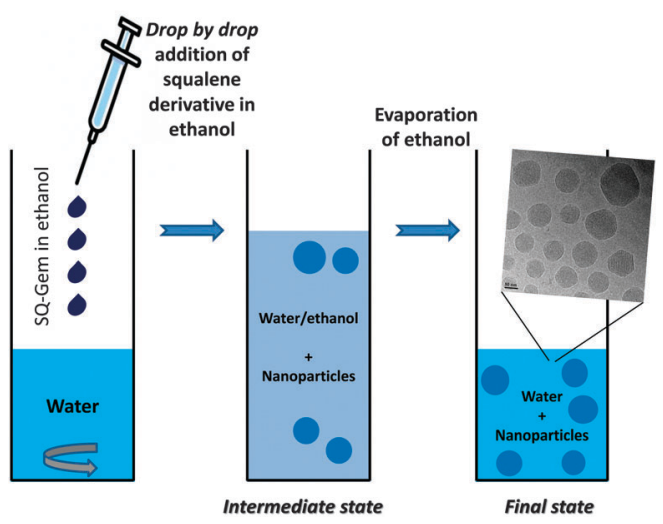

Fig. 2 Schematic representation of the two step process of squalenoyl nanoparticle formation.

solvent was then evaporated producing the final state of the nanoparticle preparation.

In a first step, a certain amount of squalenoyl conjugate (either SQ-gem, or SQ-dC, or SQ-CO2H) was properly dissolved in a suitable organic solvent, here ethanol. This solution was then added drop by drop by means of a syringe pump (PHD 2000 , Harvard Instruments) into an aqueous phase $\left(\mathrm{D}_{2} \mathrm{O}\right)$ under moderate magnetic stirring $(500 \mathrm{rpm})$. The injection rate was $3.0 \mathrm{~mL} \min ^{-1}$ for all the samples. $\mathrm{D}_{2} \mathrm{O}$ was used as the solvent instead of $\mathrm{H}_{2} \mathrm{O}$ to increase the scattering length density neutron contrast between the nanoparticles and the solvent. The progressive mixing of squalene derivated solution with $\mathrm{D}_{2} \mathrm{O}$ formed the intermediate states of the "nanomedicine" preparation. In the final step, the ethanol content was reduced to minimum by concentration using a Rotavapor around $40.0{ }^{\circ} \mathrm{C}$ and controlled vacuum (100 mbar). ${ }^{15}$ This step was repeated several times until the weight loss of the sample was equivalent to the initial amount of added ethanol used to prepare the sample. Finally, it was observed that a fraction of water or $\mathrm{D}_{2} \mathrm{O}$ co-evaporated with ethanol. This makes harder to interpret by weight measurements the exact amount of ethanol eliminated during solvent evaporation. Fortunately, the exact content of ethanol could be checked by the level of incoherent scattering (see SV, ESI $\dagger$ ). The evaporation of ethanol yielded the final state of nanoparticles which could be used for eventual pharmacological assays. ${ }^{16}$ SQ-dC nanoparticles were more stable than SQ-CO2H while SQ-gem nanoparticles displayed conditional stability. It was observed that the gemcitabine squalene intermediate states were less stable (only for a few hours), while the final state was stable up to a month, at controlled temperature (around $25.0^{\circ} \mathrm{C}$ ). It was also found that SQ-dC nanoparticles were stable from few weeks to months (by Dynamic Light Scattering (DLS), the identical size was also found after few months for the intermediate state of deoxycytidine squalene nanoparticles). The final state of the nanoparticles was prepared in the laboratory from a few days to one week before the neutron scattering experiment, while nanoparticles at intermediate states were prepared in the Chemistry laboratory of the neutron facilities (Institute Laue Langevin (ILL-Grenoble)); JCNS (Juelich Centre for Neutron Science), FRMII, Garching and Laboratory Léon Brillouin 
(LLB-Saclay), immediately before neutron scattering measurements. The final concentration of the squalenoyl derivatives is obtained by UV-Vis measurements as explained in SVII (ESI $\dagger$ ). The whole list of prepared samples is given in the SI (ESI $\dagger$ ).

\subsection{Characterization of the nanoparticles}

2.3.1 Small Angle Neutron Scattering (SANS). The range of concentration of squalene entities that could be reached by this method was rather low and Small Angle Neutron Scattering (SANS) was preferred over the more classical (and accessible) Small Angle X-ray Scattering (SAXS) to analyze the structure of these nanomaterials. ${ }^{17,18}$ Indeed, the low contrast between solvent and organic moieties was not favorable for SAXS measurements of intermediate states. In contrast, using neutrons allows a strong increase of the contrast provided that deuterated water was used as a solvent to take benefit from the large difference between hydrogen and deuterium atoms. Experimental configurations and treatment methods are presented in SII, SIV and SV (ESI $\dagger$ ).

2.3.2 Dynamic Light Scattering. DLS was carried out using a Zetasizer (MALVERN Instruments) at $20{ }^{\circ} \mathrm{C}$. All the samples used for DLS measurements were diluted in $\mathrm{D}_{2} \mathrm{O}$ by a factor of 50 to avoid multiple scattering. The DLS distribution of the size in number was reconstructed from the volume distribution yielded by the Malvern instrument analysis (used to recover the correct decrease of the autocorrelation function).

\section{Results and discussion}

\subsection{Deoxycytidine squalene nanoparticles (SQ-dC NPs)}

SANS patterns of a series of deoxycytidine squalene intermediate states with an increasing number of added drops corresponding to increasing concentrations of deoxycytidine squalene in the overall solution are presented in Fig. 3A. Even for the lowest concentration, the SANS signal from the nanoparticles was much higher than the background allowing a clear description of the sequence of events. Focusing on the high- $q$ regime first, two Bragg peaks appeared at $0.060 \AA^{-1}$ and $0.085 \AA^{-1}$ for the concentration above $0.96 \mathrm{mg} \mathrm{mL} \mathrm{m}^{-1}$. This was the signature of an internal structure of the nanoparticles from the initial steps of their formation. Ethanol was then evaporated which yields the final state. The final state at different concentrations in the overall solution is presented in Fig. 3B. At large angles, only a unique intense Bragg peak was clearly visible (at $0.09 \AA^{-1}$ ) in contrast to the intermediate stage case. This modification induced by the evaporation step supports a control of the nanoparticles' internal structure by the activity of the solvent which modifies the phase diagram of the deoxycytidine squalene-waterethanol system. On the other hand, the Bragg peak position in the final state was weakly sensitive to the deoxycytidine squalene concentration and in agreement with results previously obtained by SAXS on concentrated solution of deoxycytidine nanoparticles in water which reported the cubic structure. ${ }^{19}$ We could also confirm the cubic structure using Wide Angle X-ray Scattering (WAXS) directly on the present diluted solutions (see the inset in Fig. 3B).
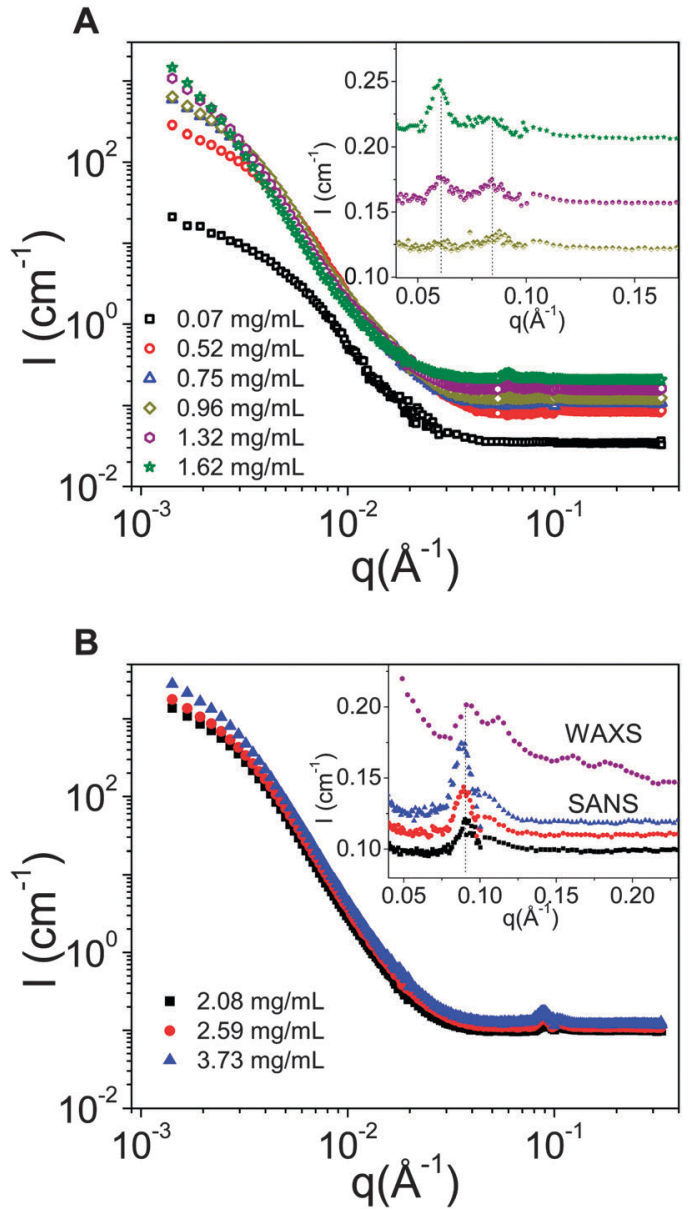

Fig. 3 (A) SANS patterns (ILL, D22) of the intermediate state of deoxycytidine squalene nanoparticles obtained during drop by drop addition of ethanol- $\mathrm{H}$ solution $\left(5.48 \mathrm{mg} \mathrm{mL}^{-1}\right.$ ) into $\mathrm{D}_{2} \mathrm{O}$. In the inset a zoom at wide angle shows the appearance and variation of a Bragg peak. (B) SANS patterns (ILL, D22) of the final state at different concentrations after evaporation. In the inset a zoom at wide angle (SANS and WAXS for the $3.73 \mathrm{mg} \mathrm{mL}^{-1}$ sample) evidences the internal structure. The concentrations (in $\mathrm{mg} \mathrm{mL}^{-1}$ ) of squalenoyl compounds in the overall solution are noted directly in the figures.

Turning to the middle $q$-range, the scattering curves offered significant information about the interfacial structure of these nanoparticles with the surrounding solvent. The signal decreased with a power law of $q^{-4}$ (called the Porod regime ${ }^{20}$ ) in the $q$ range $\sim 0.004 \AA^{-1}$ to $0.01 \AA^{-1}$ in the intermediate state and in the $q$ range $\sim 0.004 \AA^{-1}$ to $0.02 \AA^{-1}$ in the final state revealing abrupt interfaces between the nanoparticles and the solvent at the scale of a few nanometres.

At low angles, the scattering intensity saturated revealing a limited size for the structure under development in the intermediate states. However, due to a limited $q$ range $\left(q_{\exp }>\right.$ $1.410^{-3} \AA^{-1}$ ), the saturation regime was not attained and a Guinier analysis $^{21,22}$ of the signal at low $q$ could only indicate that the nanoparticles were bigger than $150 \mathrm{~nm}$ since the first drops. The higher upturn at low $q$ with concentration also revealed that particles were getting bigger.

At the final state the low- $q$ upturn of the scattering intensity was almost independent of the squalene derivative concentration. 
For the highest concentration of deoxycytidine squalene ( $C=3.73 \mathrm{mg} \mathrm{mL}{ }^{-1}$ ), a trend towards saturation was observed revealing nanoparticles of smaller size than for the intermediate states and disclosed a second effect of the evaporation of ethanol that was to reduce the size of the initially precipitated nanoparticles.

\subsection{Gemcitabine-squalene nanoparticles (SQ-gem NPs)}

Since it has been observed that nucleosides containing different head groups lead to different local organizations and in order to investigate the influence of the nucleoside moiety on the nanoparticle formation, ${ }^{19}$ we compared SQ-gem NPs to SQ-dC NPs at intermediate and final states. Unfortunately, intermediate states of SQ-gem NPs prepared in ethanol- $\mathrm{H} / \mathrm{D}_{2} \mathrm{O}$ tend to coalescence on the time scale of hours making SANS not fully reliable for long term analysis. In contrast, at the final state they displayed longer term stability, and their SANS diagrams (which were obtained in an extended $q$ range) are reported in Fig. 4 .

Focusing on the high- $q$ regime first, a Bragg peak could be traced around $0.12 \AA^{-1}$ from the lowest concentration of gemcitabine squalene (1.83 $\left.\mathrm{mg} \mathrm{mL}^{-1}\right)$. This unique Bragg peak matched quite well with the value of the second order Bragg reflection of the hexagonal phase reported in the literature for gemcitabine squalene nanoparticles. ${ }^{23}$ Additionally, the position of the Bragg peaks in the final state has been confirmed by using SAXS directly on the present diluted solutions (see the inset in Fig. 4). The absence of the first order Bragg peak was due to the presence of a minimum in the form factor of the inner pore structure.

Interestingly, in the final state, the Guinier regime below $2 \times 10^{-3} \AA^{-1}$ is still measurable for the highest concentration of SQ-gem $\left(C=3.01 \mathrm{mg} \mathrm{mL}^{-1}\right)$. Particles were bigger than for the deoxycytidine case. In contrast to the SQ-dC NPs, the low- $q$ upturn of the scattering intensity clearly depended on the concentration.

\subsection{Squalenic acid nanoparticles (SQ-CO2H NPs)}

To further extend the view of the hydrophilic head effect, we also studied the particle formation of one of the parent

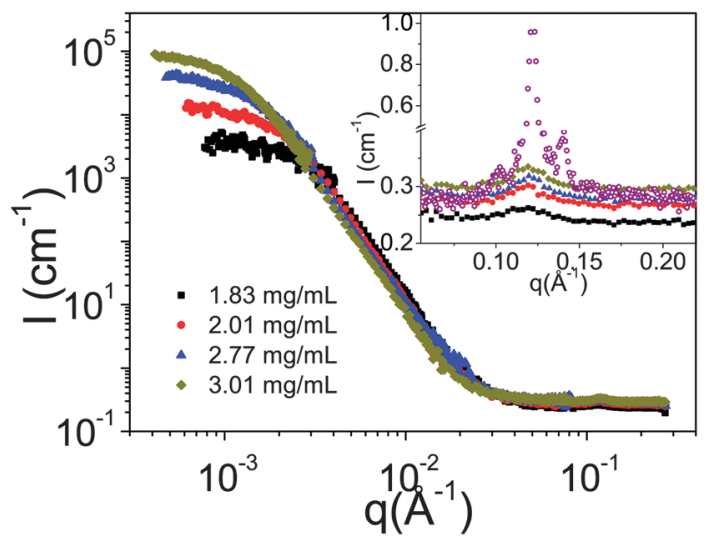

Fig. 4 SANS patterns (LLB, PAXY and TPA) of the final state of gemcitabine squalene nanoparticles at different concentrations. In the inset, a zoom at wide angle neutron (full symbol) and X-ray scattering (empty symbol) evidences the internal structure. molecules i.e. squalenic acid (SQ-CO2H), which possesses a different head group from deoxycytidine squalene and gemcitabine squalene. The results are presented in the SIII, ESI. $\dagger$

\subsection{Determination of size distribution}

A full fitting of SANS patterns was used to extract the number of particles per liter $N$, size distribution $f(r)$ and internal composition $\Delta \rho$ of SQ-dC, SQ-gem and SQ-CO2H nanoparticles. In the absence of any interaction between these nano-objects (valid approximation under the present diluted conditions), the scattering from a population of spherical objects was given by:

$$
I(q)=N \Delta \rho^{2} \int f(r) V^{2}(r) F^{2}(q, r) \mathrm{d} r
$$

where $V(r)$ and $F^{2}(r)$ were the volume and the form factor of a spherical particle of radius $r$. The matching of experimental data with eqn (1) yields $f(r)$ through $q$-dependence and $N(\Delta \rho)^{2}$ through the absolute intensity unit scaling. As shown in Fig. 5, the best result was obtained for a population of spheres with a log-normal distribution for both final and intermediate states. The fit with the $q$-dependence provided the distribution which could be compared to the one obtained by the Dynamic Light Scattering (DLS) analysis (the inset in Fig. 5A).

\subsection{Determination of swelling}

Beyond the size distribution given by $f(r)$, the full fitting allowed the extraction of the internal composition of the nanoparticles. Indeed, assuming a composition of the nanoparticles implied a value for $\Delta \rho$ (see SIV, ESI $\dagger$ ), thus a value for $N$ through $N(\Delta \rho)^{2}$. Then, the volume fraction $\Phi_{\text {tot }}$ of particles could be calculated from $f(r)$ :

$$
\Phi_{\text {tot,SANS }}=N \int f(r) V(r) \mathrm{d} r
$$

and compared to the experimentally known volume fraction $\Phi_{\mathrm{Sq}}$ of squalene derivatives added into the solution. Surprisingly, assuming that the nanoparticles were only made of squalene conjugates yielded a calculated volume fraction far too high in comparison to the added content. Hence nanoparticles had to be swollen.

Therefore, a swelling factor $\Phi_{\text {tot }}=\Phi_{\text {tot,SANs }}$ of the nanoparticles was introduced to overcome this discrepancy. The nanoparticles could be swollen either by solvent which was a mixture of water and ethanol or by water alone or by ethanol alone. $^{24}$ Assuming a type of swelling and a swelling factor $\alpha$ yielded again a specific (see SIV, ESI $\dagger$ ) $\Delta \rho$, then $N$ and finally $\Phi_{\text {tot,SANS }}$ by eqn (2). This method was effective and gave drastic results: the swelling by pure ethanol ( $\alpha$ would be below one) and the swelling by the solvent ( $\alpha$ would be beyond 20) yielded unrealistic values. Only the hypothesis of a swelling by pure water produced coherent results with $\Phi_{\text {tot }}=\Phi_{\text {tot,SANS }}$ and in the range 1-10. A first strong conclusion is thus that these nanoparticles were swollen by water.

In the case of intermediate states, the water-ethanol composition of the solvent was calculated straightforwardly from the composition of the sample. For the final states, we used the values of the incoherent background measured at large angles as explained in the SV (ESI $\dagger$ ). This revealed that even the final 

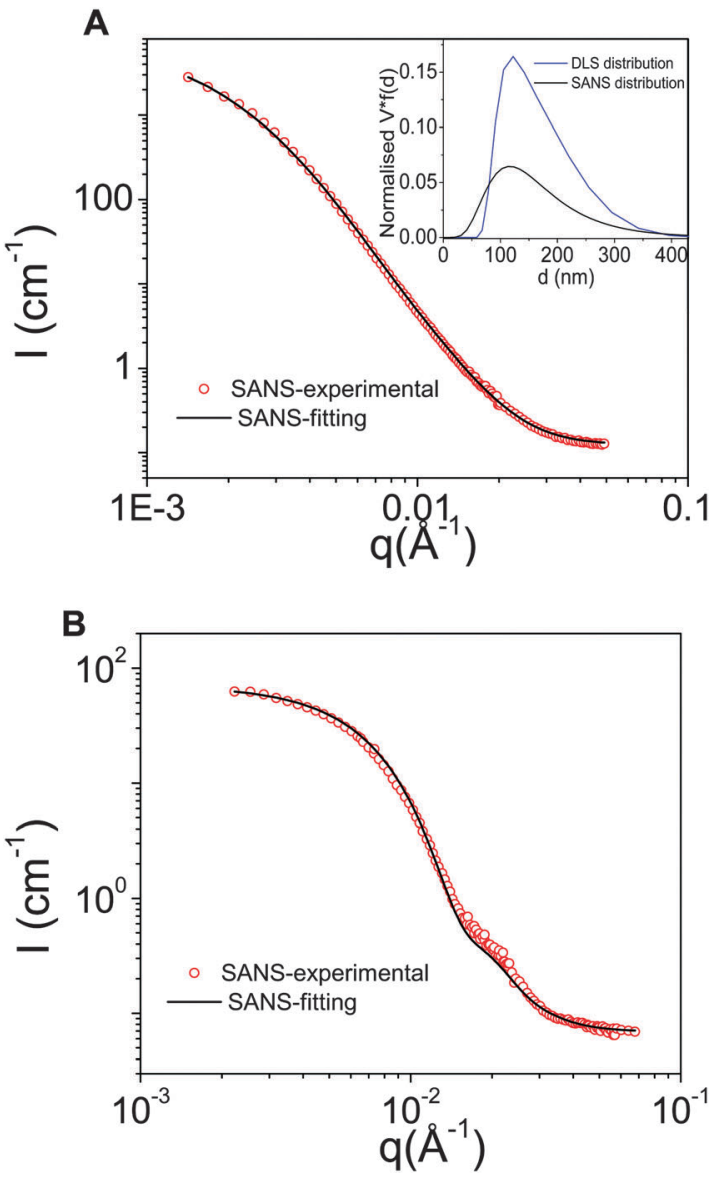

Fig. 5 (A) Fitting results of SANS intensity (ILL, D22) by eqn (1) with added incoherent background of $S Q-d C$ nanoparticles at the final state ( $C=3.73 \mathrm{mg} \mathrm{mL}^{-1}$ ). In the inset, the comparison between DLS and SANS distribution of the same sample is shown. (B) Fitting results of SANS intensity (FRM2, KWS2) by eqn (1) with the added incoherent background of $\mathrm{SQ}$-gem nanoparticles at the intermediate state $\left(C=0.41 \mathrm{mg} \mathrm{mL}^{-1}\right)$.

state did contain a large amount of ethanol and that a partial co-evaporation of water with ethanol occurred. Accordingly, we did not rely on the weight of the samples after evaporation to ascertain that ethanol has been withdrawn. ${ }^{25}$ The values of the swelling factor $\alpha$ for the three squalene derivatives versus the composition of the solvent are reported in Fig. 6. Four major conclusions can be drawn. For the intermediate and final states of deoxycytidine squalene nanoparticles, the swelling was decreasing with the addition of ethanol solution. Additionally, the swelling was between 2 and 11. It is also remarkable that in the intermediate state, SQ-CO2H nanoparticles followed the similar trend of swelling to SQ-dC. Finally, it was found that the swelling of SQ-gem NPs was lower than that of SQ-dC NPs. These findings evoke a conclusion that the swelling not only depended on the composition of the solvent but also on the chemical nature of the hydrophilic head of the conjugate. The presence of two fluorine atoms on the polar head of SQ-gem reduced the hydrophilic character of the cytidine nucleus and thus strongly influenced the packing parameter of SQ-gem with a direct consequence on the swelling. ${ }^{23}$

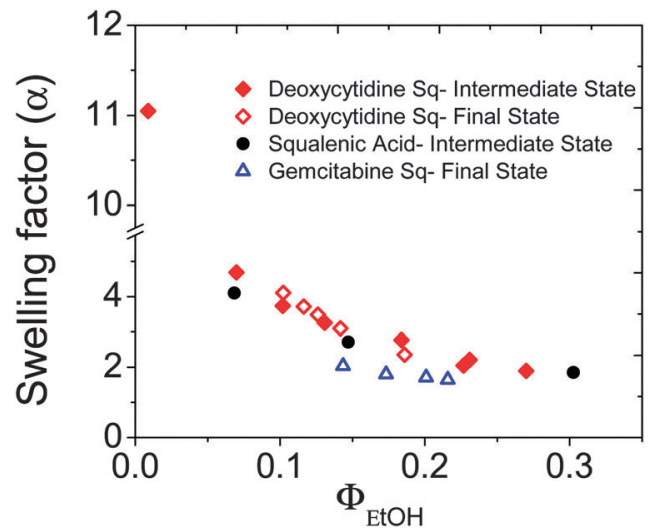

Fig. 6 Swelling factor $\alpha$ versus the ethanol fraction of the solvent for the intermediate states of $\mathrm{SQ}-\mathrm{dC}$ and $\mathrm{SQ}-\mathrm{CO} 2 \mathrm{H}$ nanoparticles and final states of $\mathrm{SQ}-\mathrm{dC}$ and $\mathrm{SQ}$-gem nanoparticles.

\subsection{Discussion on the pathway of formation}

The analysis of SANS diagrams using the water swelling model also yielded the evolution of number density and size distribution during nanoparticle formation using eqn (1). The results are reported in Fig. 7 for the three types of nanoparticles.

Along with the addition of deoxycytidine squalene ethanol drops in water (Fig. 7A), the smallest detected diameter was $42 \mathrm{~nm}$. It became bigger with the increasing number of drops. Regarding the number density of nanoparticles, a bias was that along the addition of drops, both the squalene derivative concentration and the fraction of ethanol increased. To take into account this bias and measure solely the impact imposed by the systematic gradual change of solvent, the concentration of the nano-objects was normalized by the concentration of squalenoyl derivatives (in $\mathrm{mg} \mathrm{mL}^{-1}$ ). These number densities normalized to a common concentration of $1 \mathrm{mg} \mathrm{mL}^{-1}$ (named "Norm conc.") are reported in Fig. 7 and unravel clearly that coalescence occurred for the addition of every drop since the first one. However, all the samples in the series of the intermediate states for SQ-dC were stable for days. This stability with time for a given composition of solvent implies that the coalescence occurred only during the addition of drops and the induced change of solvent. Noteworthy, the change in size was

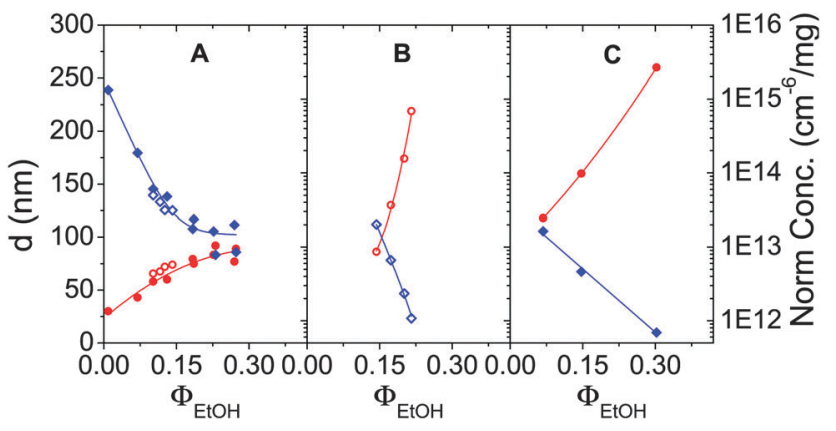

Fig. 7 Diameter in $\mathrm{nm}$ (circles) and normalized number density in $\mathrm{cm}^{-3} \mathrm{mg}^{-1}$ (diamonds) versus ethanol fraction in solvent for intermediate states (filled symbols) and final states (empty symbols). (A) SQ-dC (B) SQ-gem (C) $\mathrm{SQ}-\mathrm{CO} 2 \mathrm{H}$. 
associated with a change in solvent composition, but not with a change in concentration. ${ }^{26}$

Hence, the size was controlled by the solvent composition, which evolved with the increase of ethanol in the $\mathrm{D}_{2} \mathrm{O}$ solution. Indeed, ethanol was a lesser good solvent than water for the hydrophilic moieties of the squalenoyl conjugates. On the other hand, mobility measurements have shown that the colloidal stability of the nanoparticles was ensured by a negative zeta potential. ${ }^{27}$ This negative charge can only come from adsorbed residual charges at the surface of squalenoyl conjugate hydrophilic heads (helped in this by the strong inner swelling of the nanoparticles by water). So, the stabilization being electrostatic, when the solvent polarity was decreasing, the stability was also decreasing as shown by the colloidal stability theory. ${ }^{28}$

An intriguing question is therefore, why did the coalescence stop? Again, colloidal stability tells that when the charge was getting bigger on the objects, the stability was getting higher. Thus after a certain stage of coalescence, the global charge of the nano-objects increased and the stability was recovered.

It can therefore be concluded that the samples were at the equilibrium of swelling and internal organization, controlled by a thermodynamically intensive parameter that was the solvent composition. This was also valid for the final state samples of SQ-dC since the diameter and number values in Fig. 7 are overlapping the ones obtained for the intermediate states.

Consequently, an emerging question is the sequence of events occurring during the evaporation of ethanol. Indeed, during the addition of ethanol, a de-swelling of the sample and a gradual coalescence toward an equilibrium size were observed. Reversibly, when ethanol was evaporated from the sample, the number density of the nano-objects increased; their size was reduced, even if in the meantime they were more swollen by water. It was therefore concluded that a fragmentation of the objects occurred during the ethanol evaporation stage (Fig. 8).

Now, these conclusions were obtained for SQ-dC. But the same was also observed with SQ-gem nanoparticles in deuterated ethanol, used instead of hydrogenated ethanol which yielded more stable samples allowing the SANS measurement to be performed for the intermediate states (see SVI, ESI $\dagger$ ). A similar influence of ethanol fraction was observed for SQ-CO2H with a stronger dependence of the swelling on the ethanol content and the coalescence effect. In this case, quite big assemblies $(d=118 \mathrm{~nm})$ were formed,

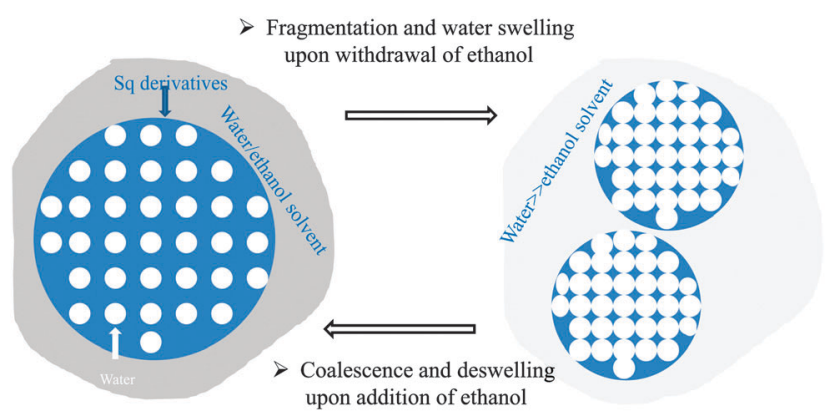

Fig. 8 Scheme of the formation of the squalenoyl nanoparticles. even at the lowest experimental concentration $\left(c=0.63 \mathrm{mg} \mathrm{mL}^{-1}\right)$ or ethanol fraction.

Finally, looking only to the final state (which is the only one used in anti-cancer treatment), it appears that gemcitabine squalene produced the best monodispersed sample. Indeed, the variances of the log-normal distribution extracted from the fits were much lower for SQ-gem nanoparticles $(0.27-0.31)$ than for SQ-dC nanoparticles (0.4-0.45). Those were also bigger in size (as reported in Fig. 7B as compared to Fig. 7A) and very sensitive to the residual amount of ethanol. However, they were still in a size range below $200 \mathrm{~nm}$ fully usable for medical applications. For SQ-CO2H nanoparticles, the size in the final states could not be extracted from the SANS fits (no Guinier regime), demonstrating that the scheme was different than in the case of deoxycytidine and gemcitabine. The influence of the "aging effect" was clearly observed for the final states of SQ-CO2H nanoparticles, pushing the particles to be aggregated and form larger nanoassemblies, which is out of the typical size range of squalenoyl prodrugs $(25-300 \mathrm{~nm})$.

Importantly, the swelling factors for the final states of SQ-gem nanoparticles were around 2. This is less than for SQ-dC nanoparticles, but still quite high which means that these efficient nanodrugs were made of $50 \%$ water. Coupling this high swelling with the hexagonal phase also means that the gemcitabine moieties, which were at the inner surface of the hexagonal channels, would be available for release upon enzymatic cleavage through these large water channels, thus inducing an efficient killing of cancer cells, as reported previously. ${ }^{29}$

\section{Conclusions}

The emerging paradigm arising from the present study is that the size obtained for these nanomedicines results from thermodynamic equilibrium, with a preferential swelling by water in a mixed water-ethanol solvent and residual charge stabilization. The important message is that the control of the solvent composition is thus of crucial importance for the size control and further scaling-up for eventual clinical translation. Noteworthily, in the most ethanol depleted solvent (final state), the nanoparticles presented a highly swollen inner structure and a size distribution below $200 \mathrm{~nm}$ well adapted for clinical applications. The direct access by large water channels to the inner content of drugs contributes to their reported improved efficiency. ${ }^{29}$

\section{Acknowledgements}

This work is supported by a public grant overseen by the French National Research Agency (ANR) as part of the "Investissements d'Avenir" program (Labex NanoSaclay, reference: ANR-10LABX-0035) in the framework of the Flagship project Nanomed for the grant supporting the position of Dr Debasish Saha and equipment. Part of the research leading to these results received funding from the European Research Council 34 (allocated to P.C.), under the European Community's Seventh Framework Programme FP7/2007-2013 (grant agreement no. 249835). 
We thank Jean-Michel Guigner from UPMC/IMPMC for providing cryo-TEM images of the solution of nanoparticles.

\section{Notes and references}

1 P. Couvreur, B. Stella, L. H. Reddy, H. Hillaireau, C. Dubernet, D. Desmaele, S. Lepêtre-Mouelhi, F. Rocco, N. Dereuddre-Bosquet, P. Clayette, V. Rosilio, V. Marsaud, J.-M. Renoir and L. Cattel, Nano Lett., 2006, 6, 2544-2548.

2 J. L. Arias, L. H. Reddy, M. Othman, B. Gillet, D. Desmaële, F. Zouhiri, F. Dosio, R. Gref and P. Couvreur, ACS Nano, 2011, 5, 1513-1521.

3 D. D. Von Hoff, T. Ervin, F. P. Arena, E. G. Chiorean, J. Infante, M. Moore, T. Seay, S. A. Tjulandin, W. W. Ma, M. N. Saleh, M. Harris, M. Reni, S. Dowden, D. Laheru, N. Bahary, R. K. Ramanathan, J. Tabernero, M. Hidalgo, D. Goldstein, E. Van Cutsem, X. Wei, J. Iglesias and M. F. Renschler, N. Engl. J. Med., 2013, 369, 1691-1703.

4 L. Harivardhan Reddy, H. Ferreira, C. Dubernet, S. Mouelhi, D. Desmaële, B. Rousseau and P. Couvreur, J. Nanopart. Res., 2008, 10, 887-891.

5 L. H. Reddy, H. Khoury, A. Paci, A. Deroussent, H. Ferreira, C. Dubernet, X. Declèves, M. Besnard, H. Chacun, S. Lepêtre-Mouelhi, D. Desmaële, B. Rousseau, C. Laugier, J.-C. Cintrat, G. Vassal and P. Couvreur, Drug Metab. Dispos., 2008, 36, 1570-1577.

6 L. H. Reddy, P.-E. Marque, C. Dubernet, S.-L. Mouelhi, D. Desmaële and P. Couvreur, J. Pharmacol. Exp. Ther., 2008, 325, 484-490.

7 L. H. Reddy, J.-M. Renoir, V. Marsaud, S. Lepetre-Mouelhi, D. Desmaële and P. Couvreur, Mol. Pharmaceutics, 2009, 6, 1526-1535.

8 S. Réjiba, L. H. Reddy, C. Bigand, C. Parmentier, P. Couvreur and A. Hajri, Nanomedicine, 2011, 7, 841-849.

9 L. Bildstein, C. Dubernet, V. Marsaud, H. Chacun, V. Nicolas, C. Gueutin, A. Sarasin, H. Bénech, S. Lepêtre-Mouelhi, D. Desmaële and P. Couvreur, J. Controlled Release, 2010, 147, 163-170.

10 S. M. Sagnella, X. Gong, M. J. Moghaddam, C. E. Conn, K. Kimpton, L. J. Waddington, I. Krodkiewska and C. J. Drummond, Nanoscale, 2011, 3, 919-924.

11 D. Desmaele, R. Gref and P. Couvreur, J. Controlled Release, 2012, 161, 609-618.
12 M. Beck-Broichsitter, E. Rytting, T. Lebhardt, X. Wang and T. Kissel, Eur. J. Pharm. Sci., 2010, 41, 244-253.

13 H. Fessi, F. Puisieux, J. P. Devissaguet, N. Ammoury and S. Benita, Int. J. Pharm., 1989, 55, R1-R4.

14 S. Schubert, J. Joseph, T. Delaney and U. S. Schubert, Soft Matter, 2011, 7, 1581-1588.

15 J. Caron, E. Lepeltier, L. H. Reddy, S. Lepêtre-Mouelhi, S. Wack, C. Bourgaux, P. Couvreur and D. Desmaële, Eur. J. Org. Chem., 2011, 2615-2628.

16 P. Couvreur, L. H. Reddy, S. Mangenot, J. H. Poupaert, D. Desmaële, S. Lepêtre-Mouelhi, B. Pili, C. Bourgaux, H. Amenitsch and M. Ollivon, Small, 2008, 4, 247-253.

17 D. Pontoni, T. Narayanan and A. R. Rennie, Langmuir, 2002, 18, 56-59.

18 B. Abécassis, F. Testard, O. Spalla and P. Barboux, Nano Lett., 2007, 7, 1723-1727.

19 E. Lepeltier, C. Bourgaux, V. Rosilio, J. H. Poupaert, F. Meneau, F. Zouhiri, S. Lepêtre-Mouelhi, D. Desmaële and P. Couvreur, Langmuir, 2013, 29, 14795-14803.

20 O. Spalla, Neutron, X-rays and Light. Scattering Methods Applied to Soft Condensed Matter, North-Holland, 1st edn, 2002.

21 A. Emmerling and J. Fricke, Proc. Third Int. Symp. Aerogels, 1992, vol. 145, pp. 113-120.

22 A. Tuteja, P. M. Duxbury and M. E. Mackay, Phys. Rev. Lett., 2008, 100, 077801.

23 E. Lepeltier, C. Bourgaux, A. Maksimenko, F. Meneau, V. Rosilio, E. Sliwinski, F. Zouhiri, D. Desmaële and P. Couvreur, Langmuir, 2014, 30, 6348-6357.

24 E. Aschenbrenner, K. Bley, K. Koynov, M. Makowski, M. Kappl, K. Landfester and C. K. Weiss, Langmuir, 2013, 29, 8845-8855.

25 R. Campardelli, G. Della Porta and E. Reverchon, J. Supercrit. Fluids, 2012, 70, 100-105.

26 J. Aubry, F. Ganachaud, J.-P. Cohen Addad and B. Cabane, Langmuir, 2009, 25, 1970-1979.

27 L. H. Reddy, C. Dubernet, S. L. Mouelhi, P. E. Marque, D. Desmaele and P. Couvreur, J. Controlled Release, 2007, 124, 20-27.

28 B. V. Derjaguin, N. V. Churaev and V. M. Muller, Surface Forces, Springer, USA, 1987, pp. 293-310.

29 L. Bildstein, V. Marsaud, H. Chacun, S. Lepetre-Mouelhi, D. Desmaele, P. Couvreur and C. Dubernet, Soft Matter, 2010, 6, 5570-5580. 